\title{
DISEÑO E IMPLEMENTACIÓN DE UN SISTEMA MÓVIL SEGUIDOR DE LÍNEA Y DETECTOR DE OBSTÁCULOS
}

\author{
Design and implementation of a follower \\ of line mobile system and the obstacles detection sensor
}

DUVANNE RAMÍREZ ${ }^{1}$, CHRISTIAN DAZA ${ }^{2}$

Recibido:20 de abril de 2018. Aceptado:15 de mayo de 2018

DOI: http://dx.doi.org/10.21017/rimci.2018.v5.n10.a52

\begin{abstract}
Resumen
Este artículo presenta la investigación, desarrollo y la aplicación de un sistema de seguidor de línea negra y detector de obstáculos, con el objetivo de analizar su comportamiento a partir de la lógica booleana en ambientes controlados, utilizando compuertas lógicas para simular inteligencia básica.

Palabras clave: Compuertas lógicas, seguidor de línea, detección, obstaculos, circuito, digital.
\end{abstract}

\section{Abstract}

This article presents the research, development and application of a black line follower and obstacle detector system, with the aim of analyzing its behavior from Boolean logic in controlled environments, using logic gates to simulate basic intelligence.

Keywords: Technology, children, learning, java, arduino, graphic interface.

\section{INTRODUCCIÓN}

Este proyecto nace para descubrir y comprender la estructura básica de un circuito digital, por medio de la creación de un vehículo seguidor de línea capaz de detenerse al detectar un obstáculo en su camino.

Con este trabajo se puede dar solución a la problemática ¿Cómo crear un cerebro electrónico capaz de seguir un indicador y tener la capacidad de detenerse ante un obstáculo?

Para lograr una efectiva respuesta a este problema se analizó e indago sobre todos los elementos y procesos que se deben tener en cuenta para crear un circuito digital (cerebro electrónico) audaz y eficaz para un carro automático.

\section{Marco teórico}

Para lograr un óptimo funcionamiento del carro se hizo una investigación acerca de cada componente necesario para el mismo:

\section{A. Sensor CNY70}

Este dispositivo es un sensor óptico infrarrojo, con un rango de corto alcance que se utiliza para detectar colores de objetos y superficies. Su uso más común es para construir pequeños robots sigue-líneas. Contiene un emisor de radiación infrarroja (fotodiodo) y un receptor (fototransistor). El fotodiodo emite un haz de radiación infrarroja, el fototransistor recibe ese haz de luz cuando se refleja sobre alguna superficie $u$ objeto [1] (ver figura 1).

1 Estudiante de Ingeniería de Sistemas de la Corporación Universitaria Republicana. Correo electrónico: christiandaza@gmail.com

2 Estudiante de Ingeniería de Sistemas de la Corporación Universitaria Republicana. Correo electrónico: yoshi2507@hotmail.com 


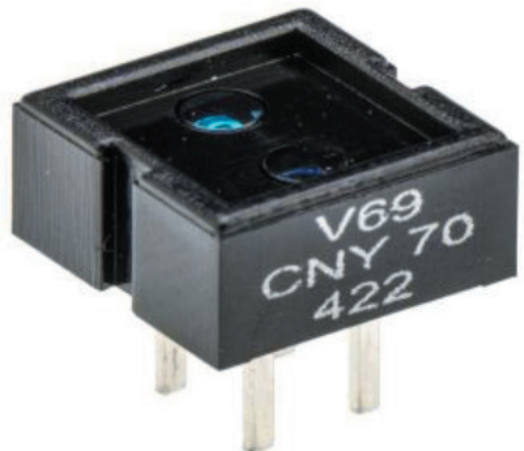

Fig.1. Sensor CNY7O.

\section{B. Sensor Digital De Obstáculos SHARP GP2Y0 D805Z0F}

GP2Y0D805Z0F es una unidad de sensor de medición de distancia, compuesto por una combinación integrada de PD (Fotodiodo), IRED (diodo emisor de infrarrojos) y circuito de procesamiento de señal [2] (ver figura 2).

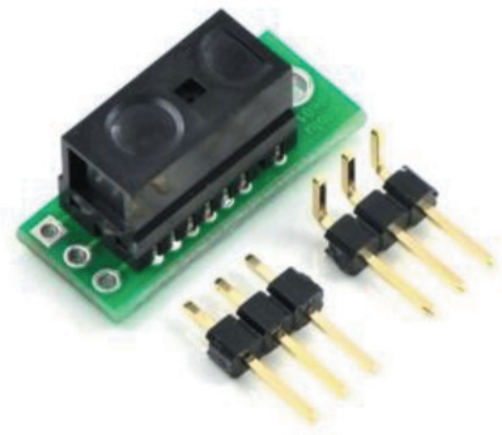

Fig. 2. SHARP GP2YOD805ZOF

\section{Compuerta Lógica 74LS04}

En lógica digital, un inversor, puerta NOT o compuerta NOT es una puerta lógica que implementa la negación lógica. A la derecha se muestra la tabla de verdad. Siempre que su entrada está en 0 (cero) o en BAJA, su salida está en 1 o en ALTA, mientras que cuando su entrada está en 1 o en ALTA, su SALIDA va a estar en 0 o en BAJA [3][4] (ver figura 3).

\section{Compuerta Lógica 74LS08}

Las compuertas AND pueden tener más de dos entradas y por definición, la salida es 1 si y solo si todas las entradas son 1 [5][6] (ver figura 4).

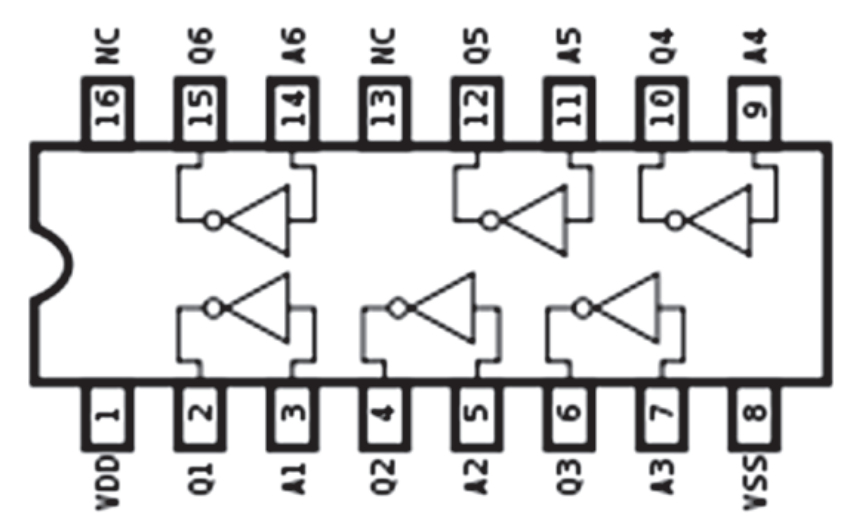

Fig. 3 Compuerta Lógica 7404.

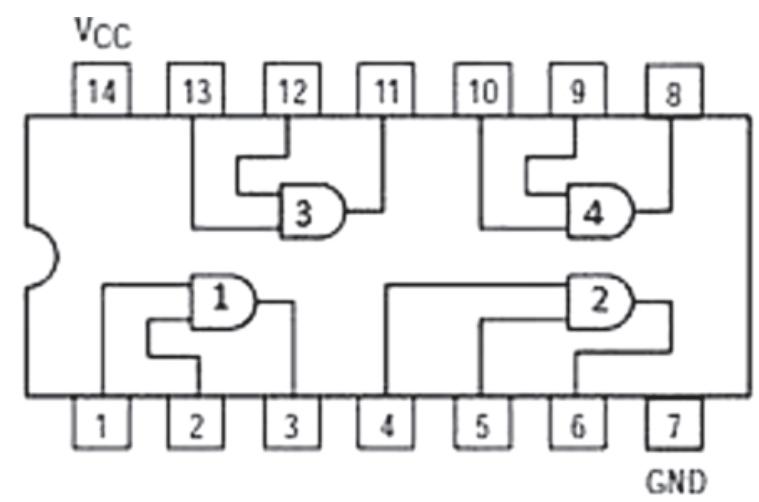

Fig. 4 Compuerta Lógica 7408

\section{E. Compuerta Lógica 74LS32}

Este circuito integrado consta de 4 puertas OR de dos entradas con salida en Tótem Pole. Su función es realizar la suma lógica de las dos variables de entrada [7][8] (ver figura 5).

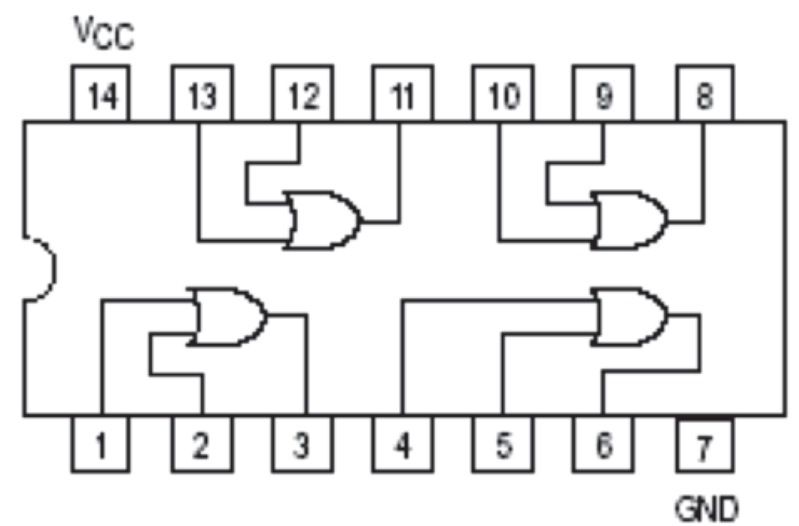

Fig. 5. Compuerta Lógica 7432 


\section{F. Potenciómetro}

Un potenciómetro son 2 resistencias conectadas en serie. A partir del nodo que se forma entre estas dos resistencias tenemos un terminal, el cual normalmente será la pata del centro en un potenciómetro de 3 patas [9] (ver figura 6).

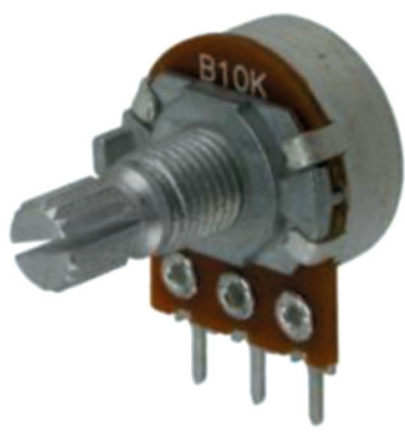

Fig. 6. Potenciómetro.

\section{G. Transistor $2 \mathrm{~N} 2222$}

Es un transistor de silicio de mediana potencia con una polaridad npn, construido mediante el proceso de base epitaxial y designado para aplicaciones de amplificación lineal y conmutación [10] (ver figura 7).

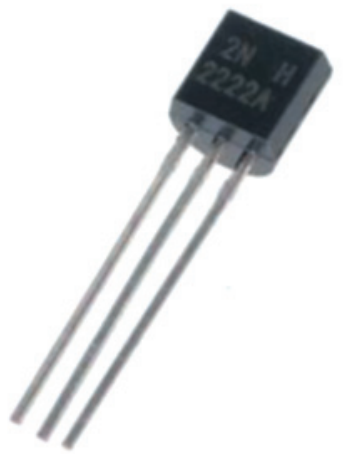

Fig. 7 Transistor 2N2222.

\section{H. Regulador LM317}

El regulador de voltaje LM317 es un dispositivo de tres pines muy fácil de utilizar. En su configuración más reducida basta con un par de resistencias para obtener el voltaje que se desee [11] (ver figura 8).

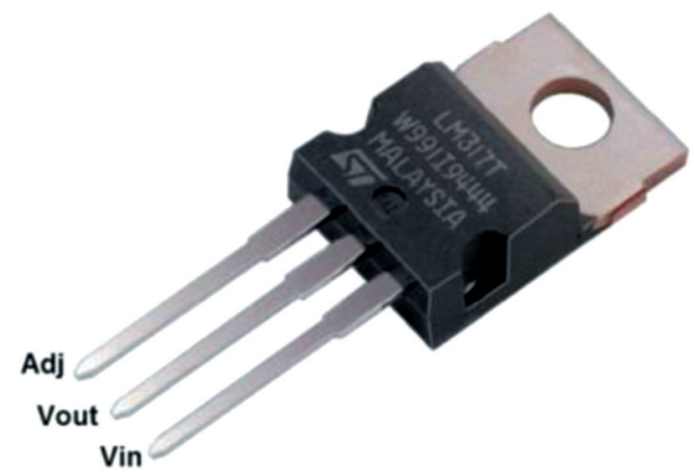

Fig. 8 Regulador LM317.

\section{Driver L293D}

Un circuito integrado de corriente continua o bipolar de pasos. Capaz de conducir corrientes bidireccionales de hasta 1 amperio en el modelo L293 y hasta $600 \mathrm{~mA}$ en el modelo L293D y con tensiones que van desde los $4.5 \mathrm{~V}$ hasta los $36 \mathrm{~V}$ en ambos modelos [12] (ver figura 9).

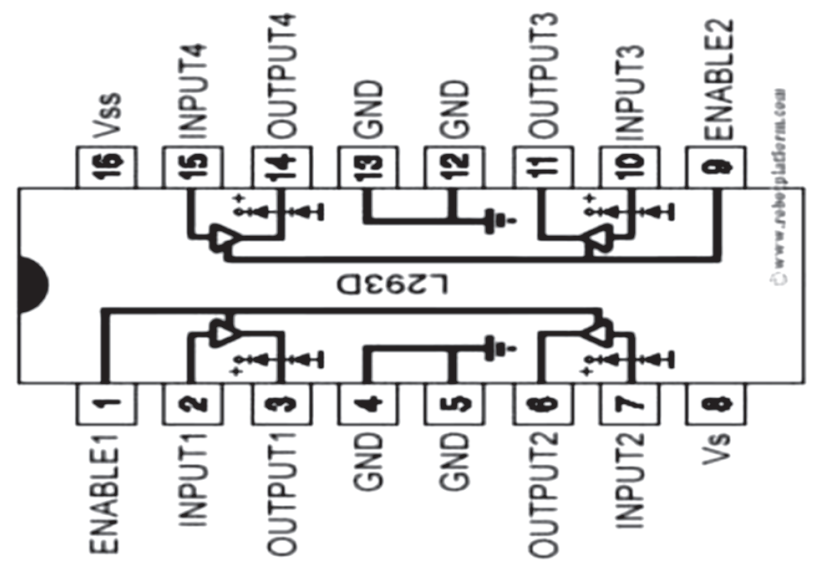

Fig. 9 Driver L293D.

\section{DESCRIPCIÓN DEL ROBOT}

Para la construcción del sistema seguidor de línea se empleo los siguientes materiales:

1. 2 Motores reductores

2. 1 Chasis en acrílico

3. 1 Driver L293d

4. 1 Regulador LM317

5. 3 Led

6. Resistencias de $1 \mathrm{k}$ ? 
7. Resistencias de $10 \mathrm{k}$ ?

8. Resistencias de 220?

9. Resistencias de 470?

10. Resistencias de 330?

11. 1 Potenciómetro de 10 k?

12.3 Transistores 2N2222A

13.2 Condensadores a 0,1 y $1 \mathrm{uF} 16 \mathrm{v}$

14.1 PCB universal

15.1Sensor GP2Y0D805Z0F/810Z0F

16.3 Sensores CNY70

17.1 Batería LiCo02 500mAh 7,4V

\section{ETAPAS DE DISEÑO}

\section{A. Diseño del chasis para el seguidor de línea}

En este aspecto se utilizo una placa de vinilo de $20 \mathrm{~cm} \times 20 \mathrm{~cm}$, también con la ayuda del software "Adobe Illustrator" se realiza un plano con la finalidad de poder realizar los cortes con una cortadora laser (ver figura 10).

\section{B. Diseño controlador de motores}

La investigación nos lleva a realizar un controlador fijo a una PCB universal para que los componentes que interactúan directamente con los motores queden de forma estática, evitando que por la manipulación del mismo los cables se desgasten y se suelten (ver figura 11 y 12).

\section{Diseño de sensores (CNY70)}

En este aspecto se investigo sobre el funcionamiento del sensor para realizar la correcta conexión de cada uno de los pines (ver figura 13).

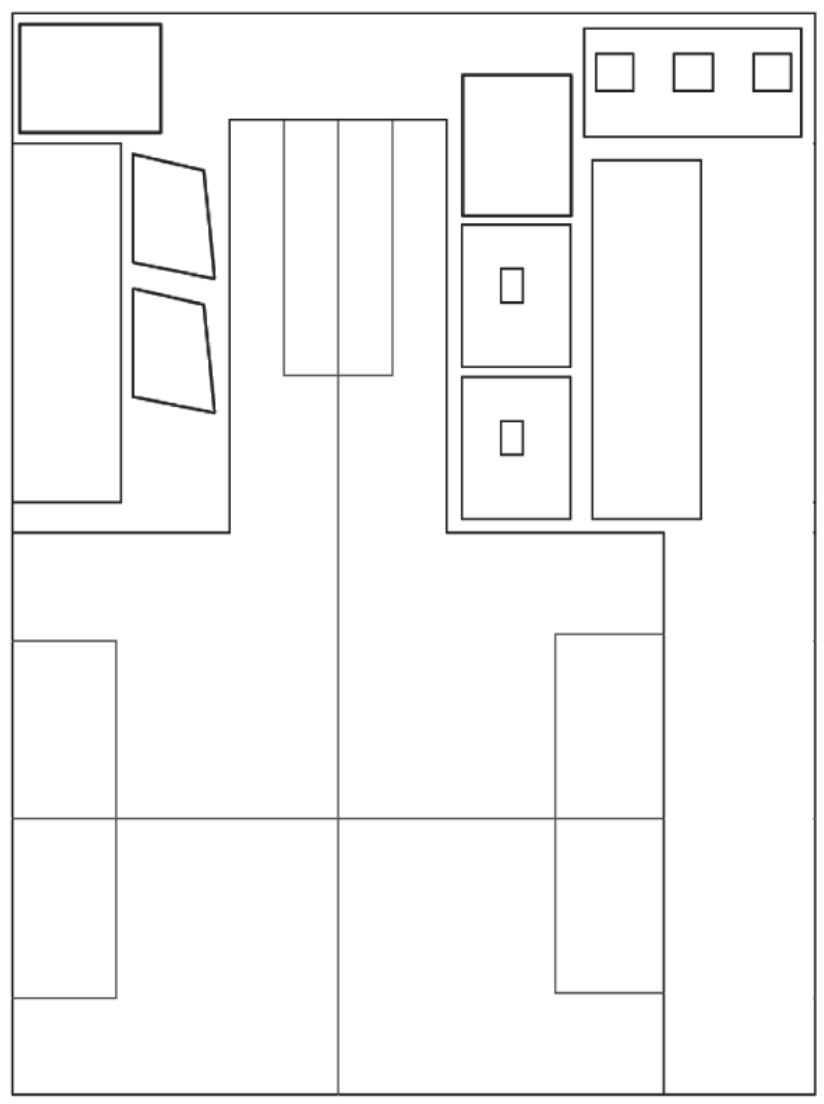

Fig. 10 Plano de cortes del chasis.

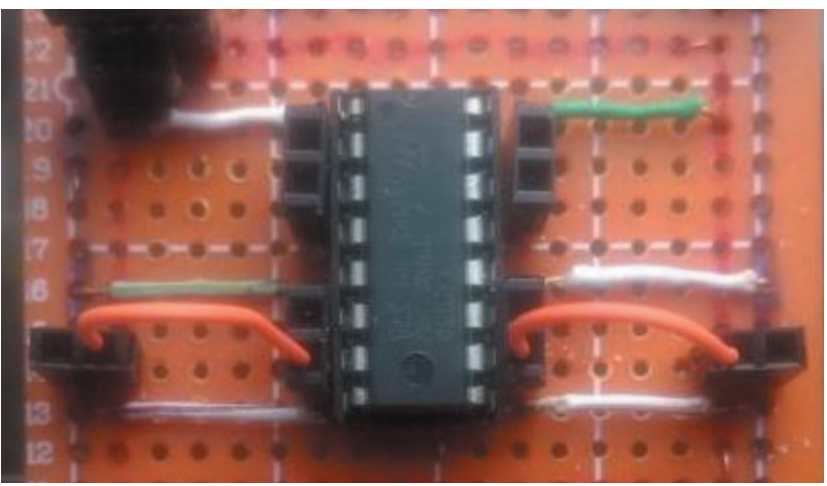

Fig. 11 Posición del cerebro con el driver L293D.

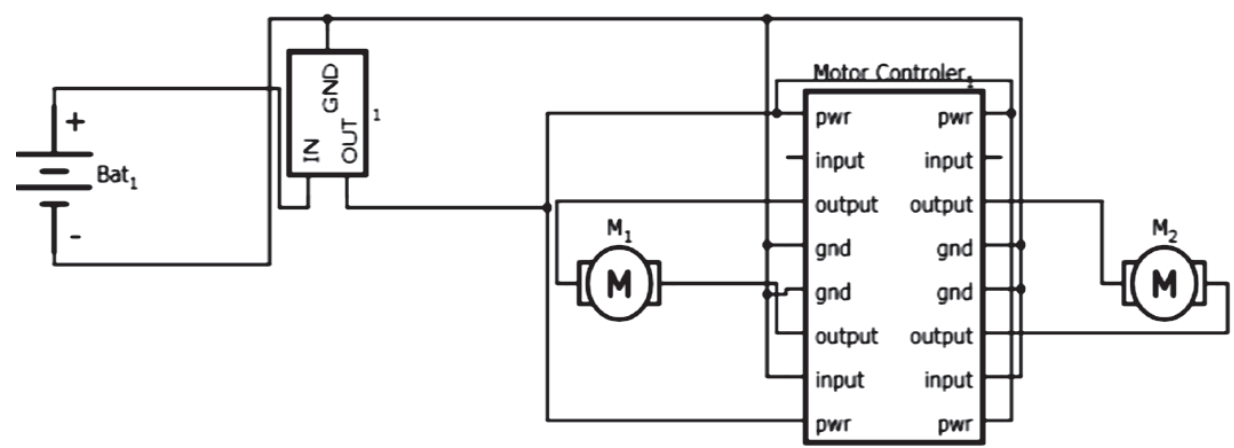

Fig. 12 Esquema PCB controlador electro-mecánico. 


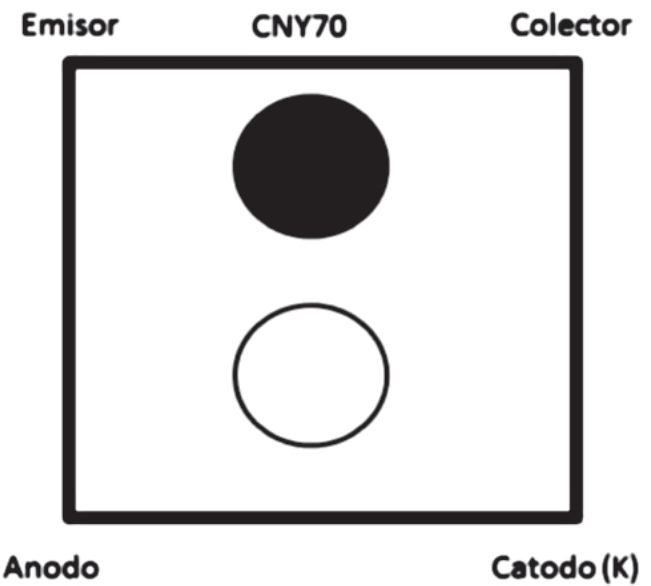

Fig. 13 Sensor CNY7O y sus convenciones.

También se utilizó un esquema de colores para identificarlos de una manera sencilla, a continuación vemos la configuración que da los mejores resultados en emisión y recolección de la información (ver figura 14 y tabla 1).

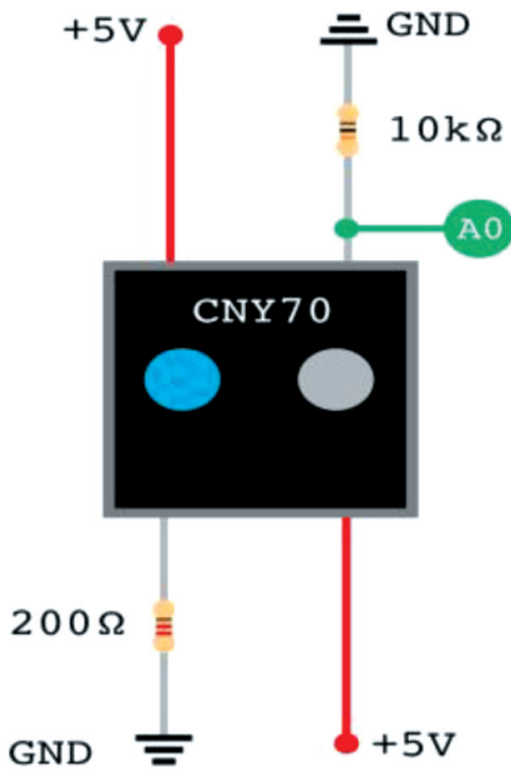

Fig. 14 Configuración Visual CNY70.

Tabla 1. Convenciones sensor CNY7O en carro.

\begin{tabular}{|l|l|l|}
\hline PIN & COLOR & DESTINO \\
\hline Colector & Café & 5V \\
\hline Emisor & Verde & $\begin{array}{l}\text { GND }(10 K \Omega) \\
\text { SALIDA }\end{array}$ \\
\hline Cátodo $(\mathrm{k})$ & Azul & GND $(220 \Omega)$ \\
\hline Ánodo & Naranja & 5V \\
\hline
\end{tabular}

Rev. Ingeniería, Matemáticas y Ciencias de la Información Vol. 5 / Núm. 10 / julio - diciembre de 2018; pág. 93-101

\section{Diseño del cerebro.}

Este aspecto se diseñó la lógica a partir de la siguiente configuración (ver figura 15).

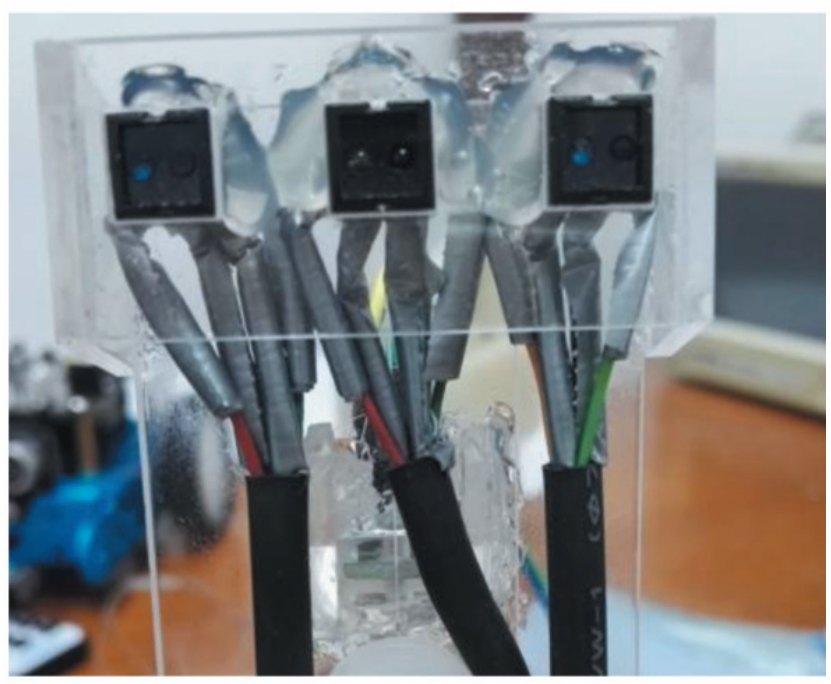

Fig. 15 Posicionamientos de los sensores.

\section{E. Tablas de verdad.}

Para crear la tabla de verdad se usó la siguiente nomenclatura:

1. OBJ: Sensor de Obstáculos.

2. IZQ: Sensor Seguidor De Línea Izquierdo.

3. CENT: Sensor Seguidor De Línea Central.

4. DER: Sensor Seguidor De Línea Derecho.

5. M. IZQ: Funcionamiento Motor Izquierdo.

6. M. DER: Funcionamiento Motor Derecho.

7. LED: Funcionamiento LED.

Con la anterior nomenclatura se logra generar una lógica con acciones complejas, que pueden hacer que un motor gire hacia cierta dirección, el carro avance e incluso pueda frenar en un sitio específico.

En conclusión a lo anterior, con esta relación se pudo formar una tabla capaz de darle un movimiento autónomo al carro, y de este modo volverlo dinámico, funcional e inteligente al manejar su lógica de un 1 a un 0 , o de manera práctica de un terreno en color blanco a un terreno en color negro. Y de este modo darle un cerebro apto para recorrer una línea negra sin salirse y a la vez de detenerse al encontrase con un obstáculo. 
La tabla de verdad que logró darle autonomía al cerebro (ver tabla 2).

\begin{tabular}{|c|c|c|c|c|c|c|} 
Tabla 2. Tabla de verdad Principal. \\
OBJ & IZQ & CENT & DER & M. IZQ & M. DER & LED \\
\hline 0 & 0 & 0 & 0 & 0 & 0 & 0 \\
\hline 0 & 0 & 0 & 1 & 1 & 0 & 0 \\
\hline 0 & 0 & 1 & 0 & 1 & 1 & 0 \\
\hline 0 & 0 & 1 & 1 & 1 & 0 & 0 \\
\hline 0 & 1 & 0 & 0 & 0 & 1 & 0 \\
\hline 0 & 1 & 0 & 1 & 1 & 1 & 0 \\
\hline 0 & 1 & 1 & 0 & 0 & 1 & 0 \\
\hline 0 & 1 & 1 & 1 & 0 & 0 & 0 \\
\hline 1 & 0 & 0 & 0 & 0 & 0 & 1 \\
\hline 1 & 0 & 0 & 1 & 0 & 0 & 1 \\
\hline 1 & 0 & 1 & 0 & 0 & 0 & 1 \\
\hline 1 & 0 & 1 & 1 & 0 & 0 & 1 \\
\hline 1 & 1 & 0 & 0 & 0 & 0 & 1 \\
\hline 1 & 1 & 0 & 1 & 0 & 0 & 1 \\
\hline 1 & 1 & 1 & 0 & 0 & 0 & 1 \\
\hline 1 & 1 & 1 & 1 & 0 & 0 & 1 \\
\hline
\end{tabular}

\section{F. Funciones lógicas}

1) Motor Izquierdo (ver tabla 3).

Tabla 3. Simplificación tabla de verdad Motor Izquierdo.

\begin{tabular}{|c|c|c|c|c|c|c|}
\hline & & & \multicolumn{4}{|c|}{$C D$} \\
\hline & & & $\begin{array}{ll}0 & 0\end{array}$ & 0 & 1 & 10 \\
\hline \multirow{4}{*}{$\mathrm{AB}$} & & 0 & 0 & 1 & 1 & 1 \\
\hline & & 1 & 0 & 1 & 0 & 0 \\
\hline & 1 & 1 & 0 & 0 & 0 & 0 \\
\hline & 1 & 0 & 0 & 0 & 0 & 0 \\
\hline
\end{tabular}

Después de realizar la simplificación por el método de karnaugh se obtiene la siguiente función.

$$
\mathrm{F} 1=(\mathrm{C}+\mathrm{D}) \cdot(\bar{B}+\bar{C}) \cdot(\bar{A})
$$

2) Motor Derecho (ver tabla 4).

Tabla 4. Simplificación tabla de verdad Motor Derecho.

\begin{tabular}{|c|c|c|c|c|c|c|}
\hline & & & \multicolumn{4}{|c|}{$C D$} \\
\hline & & & $\begin{array}{ll}0 & 0\end{array}$ & $\begin{array}{ll}0 & 1\end{array}$ & $\begin{array}{ll}1 & 1\end{array}$ & 10 \\
\hline \multirow{4}{*}{$\mathrm{AB}$} & 0 & 0 & 0 & 0 & 0 & 1 \\
\hline & 0 & 1 & 1 & 1 & 0 & 1 \\
\hline & 1 & 1 & 0 & 0 & 0 & 0 \\
\hline & 1 & 0 & 0 & 0 & 0 & 0 \\
\hline
\end{tabular}

Después de hacer la simplificación por el método de karnaugh se obtiene la siguiente función.

$$
\mathrm{F} 2=(\mathrm{B}+\mathrm{C}) \cdot(\bar{C}+\bar{D}) \cdot(\bar{A})
$$

\section{3) Led}

\begin{tabular}{|c|c|c|c|c|c|c|}
\hline & & & \multicolumn{4}{|c|}{$C D$} \\
\hline & & & $\begin{array}{ll}0 & 0\end{array}$ & 0 & 1 & $\begin{array}{ll}10 \\
1\end{array}$ \\
\hline \multirow{4}{*}{$\mathrm{AB}$} & & & 0 & 0 & 0 & 0 \\
\hline & & & 0 & 0 & 0 & 0 \\
\hline & & & 1 & 1 & 1 & 1 \\
\hline & & 0 & 1 & 1 & 1 & 1 \\
\hline
\end{tabular}

Tabla 5. Simplificación tabla de verdad Led.

Después de hacer la simplificación por el método de karnaugh se obtiene la siguiente función.

$$
\mathrm{F} 3=\mathrm{A}
$$

Realizando las simplificaciones se puede ver que estamos utilizando un total de 12 compuertas distribuidas de la siguiente forma:

4 NOT

$4 \mathrm{OR}$

4 AND

\section{G. Circuito Lógico}

\section{Motor Izquierdo}

El juego cuenta con 2 clases que se encargan de la parte de los datos del usuario y 13 Jframe. El

Rev. Ingeniería, Matemáticas y Ciencias de la Información Vol. 5 / Núm. 10 / julio - diciembre de 2018; pág. 93-101 
principal es donde el usuario ingresa para entrar al menú principal, si este no está registrado o no se quiere registrar no puede ingresar al juego (ver figura 16).

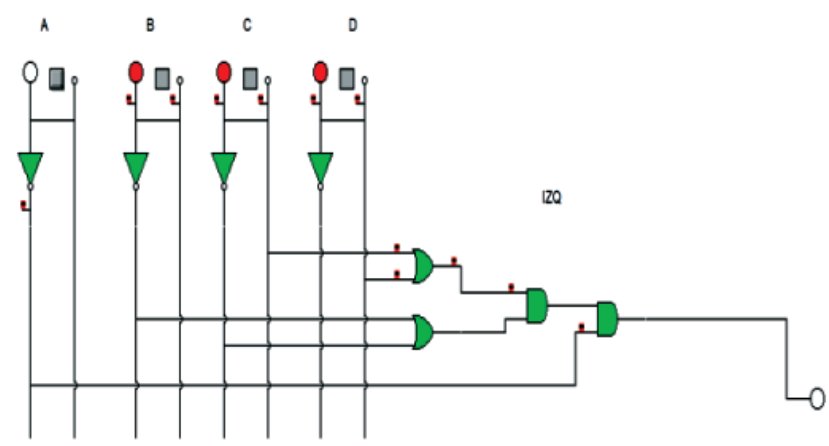

Fig. 16 Esquema Lógico Motor Izquierdo.

\section{Motor Derecho (ver figura 17).}

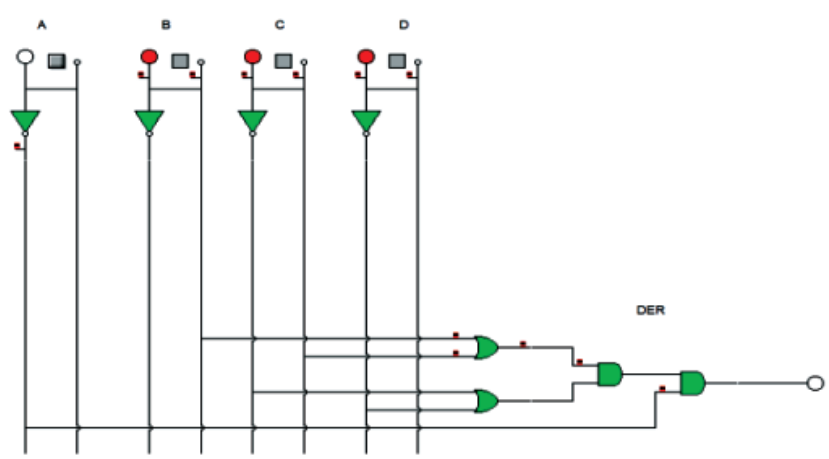

Fig. 17.Esquema Lógico Motor Derecho.

\section{Simulaciones}

Las siguientes simulaciones muestran que la lógica implementada es correcta y funcional para el prototipo físico, de esta forma se garantiza que la implentación física del seguidor no tenga errores (ver figura 18 a 20).

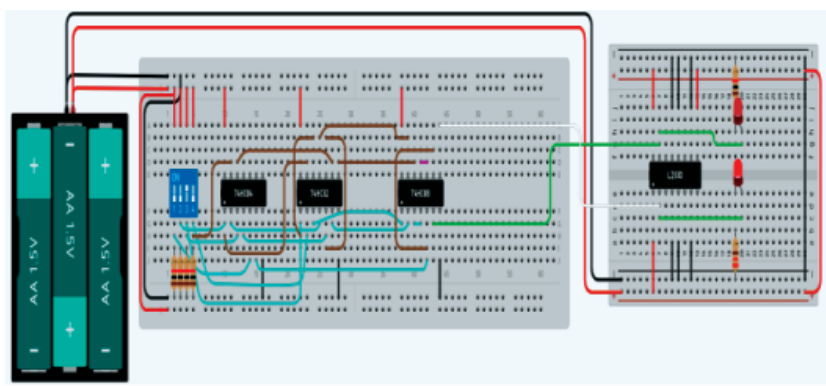

Fig. 18. Simulación circuito en función al movimiento de los dos motores.

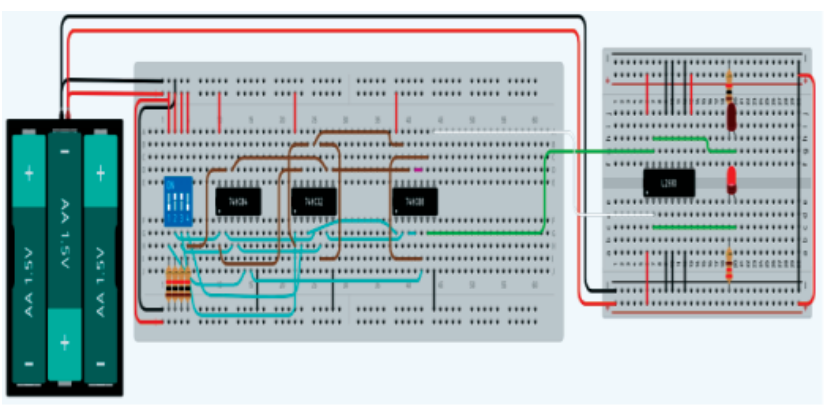

Fig. 19. Simulación circuito en función al movimiento del motor derecho.

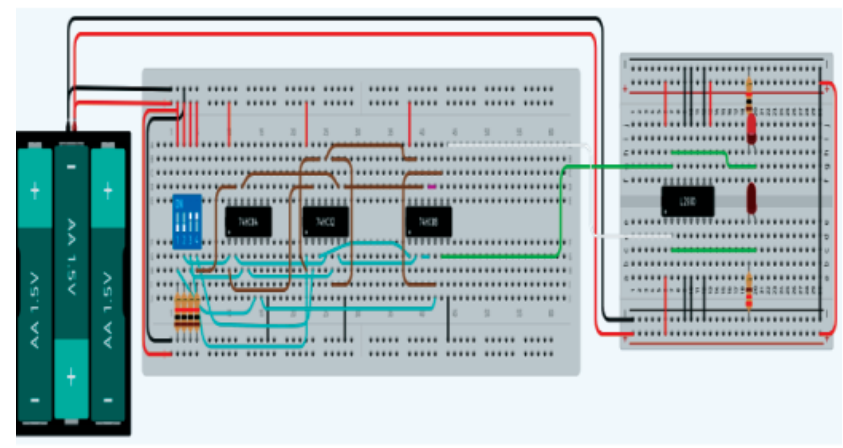

Fig. 20. Simulación circuito en función al movimiento del motor Izquierdo.

\section{Resultados obtenidos}

\section{A. Cableado Final Del Driver L293D.}

En la siguiente imagen se evidencian todas las conexiones necesarias para el perfecto funcionamiento del Driver L293D las cuales son (ver figura 21).

- Conexión de los 2 Motores.

- Conexión de los 3 Sensores

- Conexión de Voltaje.

- Conexiones a Tierra.

\section{B. Compuertas lógicas}

En esta imagen se ven las relaciones entre compuertas, las cuales le dan autonomía al carro, los componentes que se ven son los siguientes (ver figura 22).

- Compuerta Negadora 74LS04.

- Compuerta De Productos 74LS08.

- Compuerta De Sumas 74LS32.

- 2 Led's Rojos 


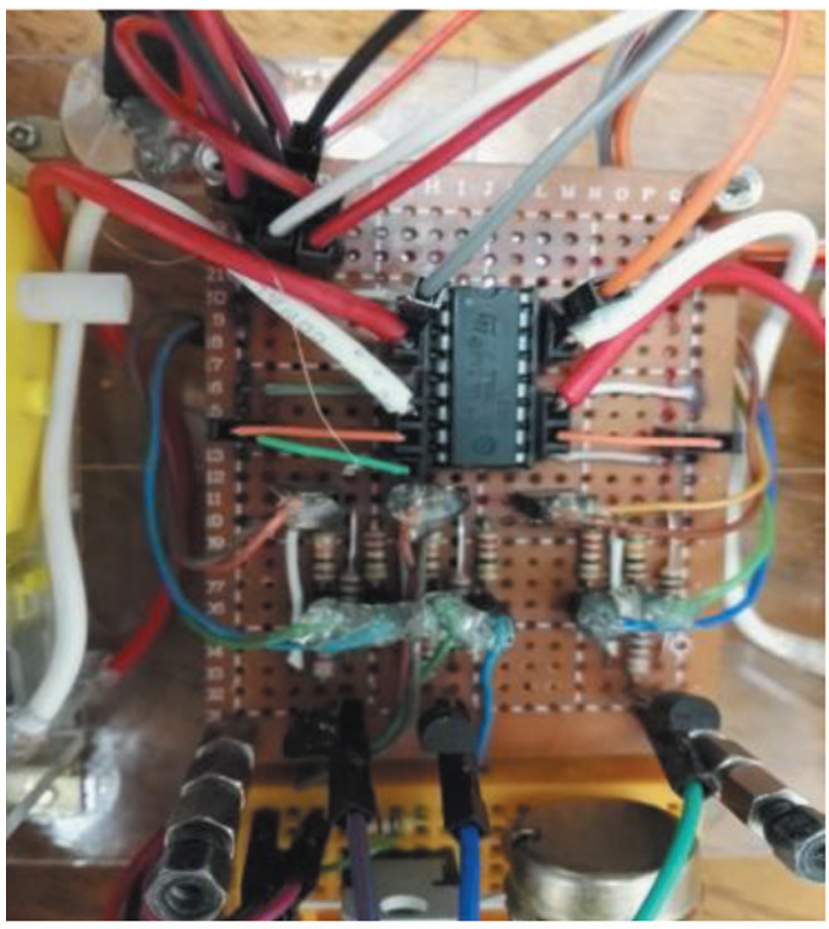

Fig. 21. Implementación Del Driver L293D.

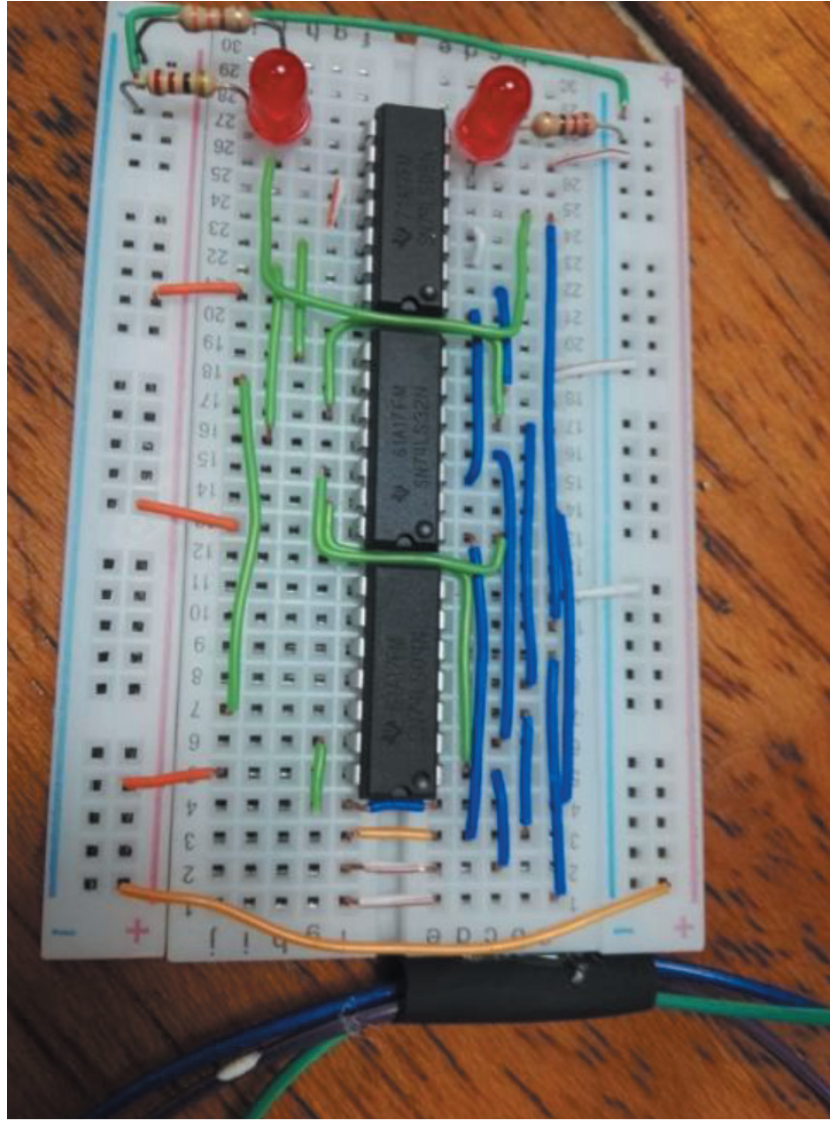

Fig. 22. Autonomía y complejidad del carro.

\section{SHARP GP2Y0D805Z0F y Sensores CNY70}

En la imagen que sigue se ve el posicionamiento del sensor de obstáculos con los 3 sensores seguidores de línea (ver figura 23).

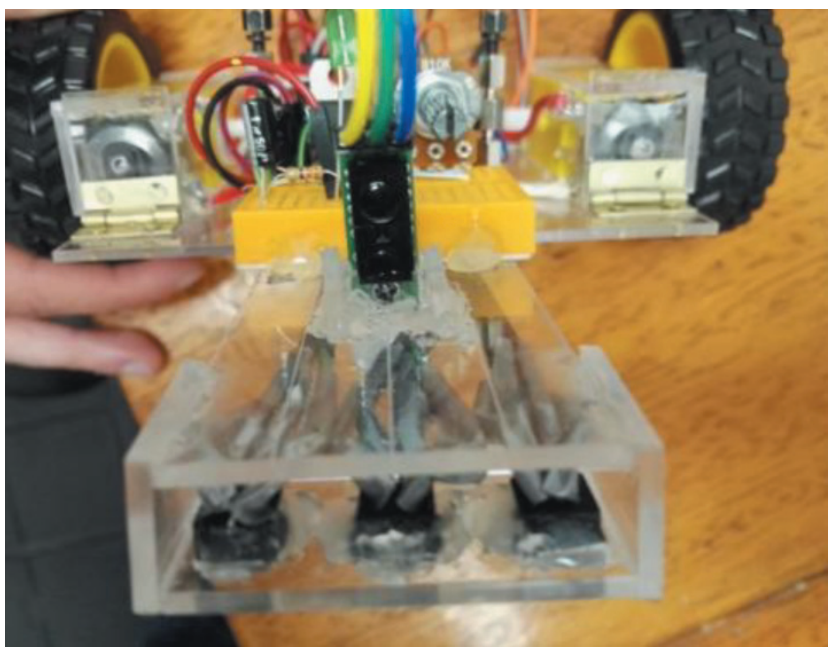

Fig. 23. Posicionamiento de los sensores.

\section{Cableado De Motores y Fuente De Voltaje}

En esta próxima imagen se visualizan los motores conectados debidamente al driver L293D y donde quedan las salidas que se conectan a una fuente de voltaje (Batería, Cargador, etc.) (ver figura 24).

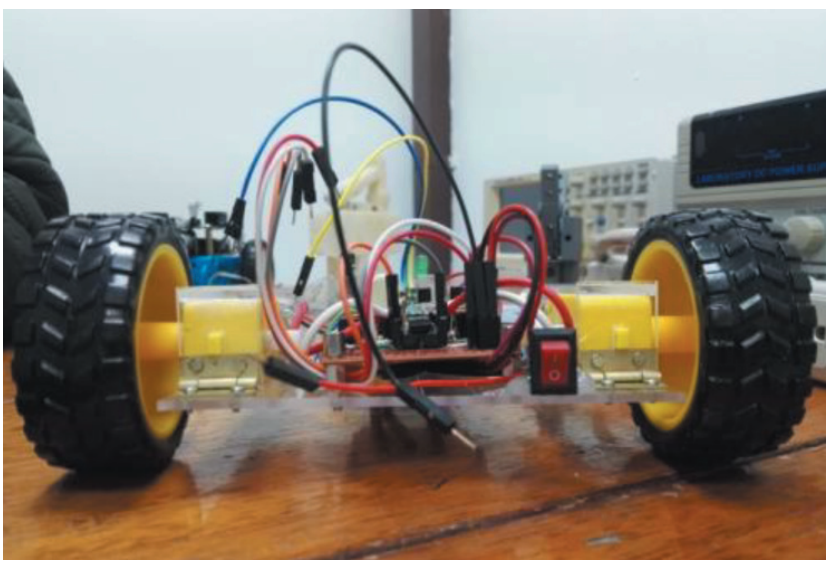

Fig. 24 Motores y Fuente.

\section{E. Carro final}

En la última imagen se pueden ver todos los componentes del carro en perfecta posición, y a la vez dándole el más eficaz y óptimo funcionamiento realizado en esta investigación (ver figura 25). 


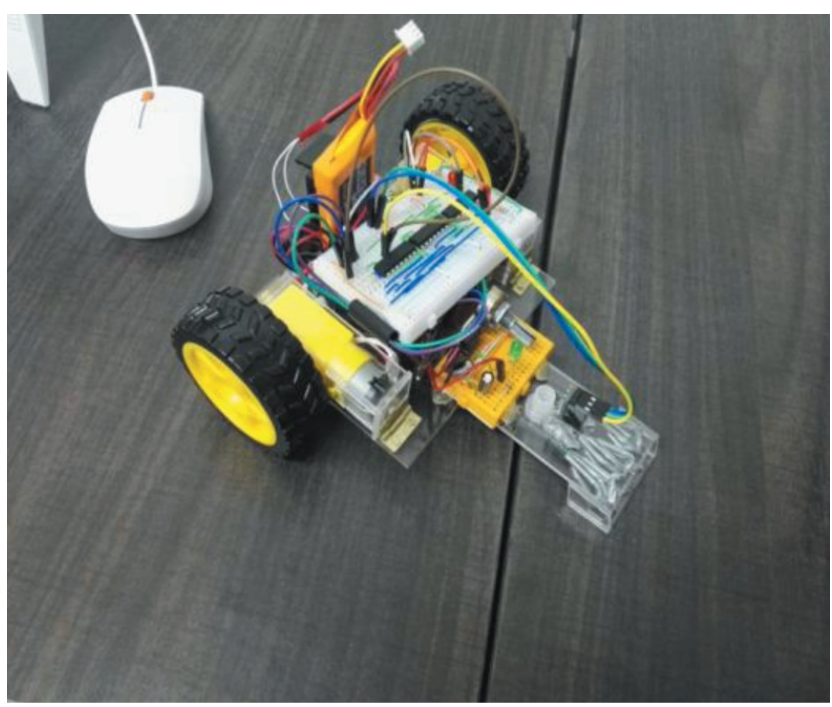

Fig. 25. Prototipo Final.

\section{CONCLUSIONES}

Para concluir este trabajo podemos evidenciar que para llegar a la solución de este problema se necesitó investigar sobre cada componente del circuito como los son sensores, transistores, resistencias, potenciómetros, led's y cada una de las compuertas necesarias para la solución óptima de este proyecto, de este modo también se solucionó cada uno de los inconvenientes físicos y lógicos del carro como lo fueron:

- El chasis fue algo trascendental en la solución y eficacia en la creación de este carro, porque con este se pudieron acomodar adecuadamente cada uno de los elementos del modelo funcionando cada uno perfectamente sin afectarse entre sí.

- La organización de la implementación de cada componente en la protoboard, dándole estilo y una fácil comprensión del cableado en el circuito.

- El voltaje y la corriente fueron unas de las variables más importantes para que el sistema electrónico y en especial el sensor digital detector de objetos funcionaran adecuadamente, ya que de estos dependían el funcionamiento de los motores, sensores de línea y que el detector de objetos lograra frenar al detectar un obstáculo.
Este tipo de trabajos son muy dinámicos y prácticos que hacen que nosotros como estudiantes exploremos y aprovechemos más de nuestras capacidades físicas y mentales frente a la solución de cualquier situación o circunstancia que se nos presente, logrando superar estas dificultades de manera eficaz y perspicaz dando un mejor desempeño de uno mismo.

\section{REFERENCIAS}

[1] Tecnosefarad. El blog de Tecnología del IES Sefarad (Toledo). Disponible en: http://www.tecno sefarad.com/2014/03/sensor-de-infrarrojoscny70-como-entrada-digital/. 2014.

[2] Pololulu. Sharp Corporation [Internet]. Disponible en: https://www.pololu.com/file/0J284/GP2 Y0D805Z0F.pdf. 2016.

[3] Fletcher, William (1980). An engineering approach to digital design (en inglés). Prentice-Hall. p. 78. ISBN 0-13-277699-5.

[4] P. García, Compuertas Lógicas (Electrónica Digital) NAND, NOT [Internet]. Disponible en: https:/ / www.youtube.com/watch?v=7gJqFfW7034. 2015.

[5] Ecured, Circuitos [Internet]. Disponible en: https:/ /www.ecured.cu/Circuito_integrado_7408. 2013.

[6] P. García, Curso Electrónica Digital Básica - Compuertas lógicas (AND) [Internet]. Disponible en: https://www.youtube.com/watch?v=O9Dty Hnp5aI. 2015.

[7] Electronica-teoriaypractica, Electrónica: teoría y práctica [Internet]. Disponible en: http:/ / electronica-teoriaypractica.com/circuito-7432-ttl/. 2011.

[8] P. García, Compuerta OR, circuito para comprobar la tabla de verdad [Internet]. Disponible en: https:/ / www.youtube.com/watch?v=9owIr0Uc3bI. 2015.

[9] A. García González, Electrónica [Internet]. Disponible en: http:/ / panamahitek.com/que-es-y-comofunciona-un-potenciometro/. 2016.

[10] Ecured. Circuitos [Internet]. Disponible en: https:/ /www.ecured.cu/Transistor_2N2222

[11] Enrique Educachip, Regulador [Internet]. Disponible en: http:/ / www.educachip.com/reguladorde-voltaje-lm317/. 2014.

[12] M. Villasur, Driver L293D [Internet]. Disponible en: http:/ / www.manuelvillasur.com/2012/10/driver-1293d-de-texas-instruments.html. 2012. 
